TRANSACTIONS OF THE

AMERICAN MATHEMATICAL SOCIETY

Volume 353, Number 11, Pages 4529-4544

S 0002-9947(01)02813-6

Article electronically published on June 1, 2001

\title{
PERIODIC SOLUTIONS OF CONSERVATION LAWS CONSTRUCTED THROUGH GLIMM SCHEME
}

\author{
HERMANO FRID
}

\begin{abstract}
We present a periodic version of the Glimm scheme applicable to special classes of $2 \times 2$ systems for which a simplication first noticed by Nishida (1968) and further extended by Bakhvalov (1970) and DiPerna (1973) is available. For these special classes of $2 \times 2$ systems of conservation laws the simplification of the Glimm scheme gives global existence of solutions of the Cauchy problem with large initial data in $L^{\infty} \cap B V_{l o c}(\mathbb{R})$, for Bakhvalov's class, and in $L^{\infty} \cap B V(\mathbb{R})$, in the case of DiPerna's class. It may also happen that the system is in Bakhvalov's class only at a neighboorhood $\mathcal{V}$ of a constant state, as it was proved for the isentropic gas dynamics by DiPerna (1973), in which case the initial data is taken in $L^{\infty} \cap B V(\mathbb{R})$ with $\mathrm{TV}\left(U_{0}\right)<$ const., for some constant which is $O\left((\gamma-1)^{-1}\right)$ for the isentropic gas dynamics systems. For periodic initial data, our periodic formulation establishes that the periodic solutions so constructed, $u(\cdot, t)$, are uniformly bounded in $L^{\infty} \cap B V([0, \ell])$, for all $t>0$, where $\ell$ is the period. We then obtain the asymptotic decay of these solutions by applying a theorem of Chen and Frid in (1999) combined with a compactness theorem of DiPerna in (1983). The question about the decay of Nishida's solution was proposed by Glimm and Lax in (1970) and has remained open since then. The classes considered include the $p$-systems with $p(v)=\gamma v^{-\gamma},-1<\gamma<+\infty, \gamma \neq 0$, which, for $\gamma \geq 1$, model isentropic gas dynamics in Lagrangian coordinates.
\end{abstract}

\section{Introduction}

The purpose of this paper is the construction of globally defined periodic entropy solutions of systems of conservation laws using a periodic version of the Glimm scheme. Such a periodic version for the Glimm scheme is not possible in general, if one has to follow the same procedures originally introduced by Glimm [9] in order to prove the uniform boundedness of the total variation of the approximate solutions. The main obstruction is that the functional used by Glimm contains a quadratic part, consisting of products of strengths of approaching waves, which has no periodic equivalent: waves that are approaching in a certain interval of one period may not be approaching in another interval of one period. In other words, in the cylinder $S^{1} \times[0, \infty)$, any wave is approaching to any other wave of a different family. On the other hand, the notion of approaching waves in the whole line seems to be essential in the process of bounding the effects of wave interactions, in general. Nevertheless, as we describe below, starting with Nishida [15], there have been many

Received by the editors August 1, 2000 and, in revised form, November 29, 2000.

1991 Mathematics Subject Classification. Primary 35L65; Secondary 35B35, 76N15.

Key words and phrases. Glimm's scheme, periodic solutions, decay of periodic solutions, conservation laws. 
$2 \times 2$ systems found, and even some very special $n \times n$ systems, with $n>2$, for which it is possible to prove the uniform boundedness of the total variation of the approximate solutions using functionals which are linear in the wave strengths, that is, which contain no products of wave strengths. These classes include the systems of isentropic gas dynamics. For them, a periodic version of the Glimm scheme is then possible as we will show. This relatively simple observation allows one to construct globally defined uniformly bounded periodic entropy solutions, which in many cases was not known. Then, using a theorem in 2] combined with a compactness theorem in [8], one can prove the decay of these solutions, thus solving some important open problems. We next pass to a more precise description of our results.

In 15, Nishida proved the global existence of weak solutions of the Cauchy problem for the system

$$
\begin{aligned}
& v_{t}-u_{x}=0, \\
& u_{t}+(1 / v)_{x}=0,
\end{aligned}
$$

constructed by an adaptation of Glimm's method, for any bounded initial data belonging to $B V_{l o c}(\mathbb{R})$, assuming values in the region $v>\delta>0$. According to Nishida's theorem, the $L^{\infty}$ norm of these weak solutions may increase unboundedly with time. His result was later extended by Bakhvalov [1], who identified a class of $2 \times 2$ systems

$$
U_{t}+F(U)_{x}=0,
$$

with $U=\left(u_{1}, u_{2}\right), F(U)=\left(f_{1}(U), f_{2}(U)\right)$, to which Nishida's reasoning could be applied. The systems in the class determined by Bakhvalov are characterized by some conditions satisfied by the image of the corresponding shock curves in a plane of Riemann invariants. Here we will not give a description of Bakhvalov's conditions but instead we will consider the main representative of this class:

$$
\left\{\begin{array}{l}
v_{t}-u_{x}=0, \\
u_{t}+p(v)_{x}=0,
\end{array}\right.
$$

where $p$ is a smooth function defined in $v>0$ satisfying

$$
p^{\prime}(v)<0, \quad p^{\prime \prime}(v)>0, \quad 3\left(p^{\prime \prime}(v)\right)^{2} \leq 2 p^{\prime}(v) p^{\prime \prime \prime}(v), \quad \text { for } v>0 .
$$

For instance, $p(v)=\gamma v^{-\gamma}$, with $-1<\gamma \leq 1, \gamma \neq 0$. It is not difficult to show that if $p_{1}$ and $p_{2}$ satisfy (1.3), then $C_{1} p_{1}+C_{2} p_{2}$ satisfies (1.3), for any pair of nonnegative constants $\left(C_{1}, C_{2}\right) \neq(0,0)$.

Set $U=(v, u)$ and

$$
z(U) \equiv u+\int^{v} \sqrt{-p^{\prime}(v)} d v, \quad w(U) \equiv u-\int^{v} \sqrt{-p^{\prime}(v)} d v .
$$

Consider the initial condition

$$
U(x, 0)=U_{0}(x), \quad x \in \mathbb{R},
$$

with

$$
0<\delta \leq v_{0}(x) \leq M, \quad\left|u_{0}(x)\right| \leq M,
$$

for some positive constants $\delta, M$. Suppose that

$$
\operatorname{TV}\left(U_{0} \mid[0, \ell)\right)<\infty,
$$


and

$$
U_{0}(x+\ell)=U_{0}(x)
$$

If

$$
\Phi(v)=-\int^{v} \sqrt{-p^{\prime}(v)} d v \nrightarrow+\infty, \quad \text { as } v \rightarrow 0,
$$

which is the case, for instance, when $p(v)=\gamma v^{-\gamma}$, with $-1<\gamma<1, \gamma \neq 0$, we further assume that

$$
w_{0}=\sup _{x \in \mathbb{R}} w\left(U_{0}(x)\right)<z_{0}=\inf _{x \in \mathbb{R}} z\left(U_{0}(x)\right) .
$$

In this paper we prove the following theorem concerning problems (1.2)-(1.8). Let

$$
\bar{U}=\frac{1}{\ell} \int_{0}^{\ell} U_{0}(x) d x .
$$

Theorem 1.1. There exists a global periodic entropy solution of (1.2)-(1.10), which belongs to $L^{\infty} \cap B V_{\text {loc }}\left(\mathbb{R} \times \mathbb{R}_{+}\right)$. This solution $U(x, t)$ also satisfies $w(U(x, t))$ $\leq w_{0}, z(U(x, t)) \geq z_{0}$. Furthermore, it has the following decay property:

$$
\text { ess } \lim _{t \rightarrow \infty} \int_{0}^{\ell}|U(x, t)-\bar{U}| d x=0 .
$$

We recall that the problem of the decay of the large data solutions obtained by Nishida was proposed in [10] and has remained open since then. On passing from Lagrangian to Eulerian coordinates, Theorem 1.1 gives a global periodic entropy solution in $L^{\infty} \cap B V_{\text {loc }}\left(\mathbb{R} \times \mathbb{R}_{+}\right)$of the corresponding problem for the system

$$
\left\{\begin{array}{l}
\rho_{t}+(\rho u)_{x}=0, \\
(\rho u)_{t}+\left(\rho u^{2}+P(\rho)\right)_{x}=0,
\end{array}\right.
$$

where $\rho=v^{-1}$ and $P(\rho)=p(1 / \rho)$.

An important fact concerning Bakhvalov's conditions was noticed by DiPerna [7]. Namely, he proved that if in (1.2) one has $p^{\prime}(v)<0, p^{\prime \prime}(v)>0$, for $v>0$, and

$$
\lim _{v \rightarrow 0} v^{\alpha+j} \frac{d^{j}}{d v^{j}} p(v) \neq 0, \quad \lim _{v \rightarrow \infty} v^{\beta+j} \frac{d^{j}}{d v^{j}} p(v) \neq 0, \quad \alpha, \beta>1, \quad j=0, \ldots, 5,
$$

then, given any $\bar{U} \in\{(u, v): v>0\}$, there exist Riemann invariants, $z^{\prime}=\phi(z)$, $w^{\prime}=\psi(w)$, and a neighborhood $\mathcal{V}=\mathcal{V}(\bar{U})$ such that the image in the $\left(z^{\prime}, w^{\prime}\right)$-plane of the segments of shock curves contained in $\mathcal{V}$ satisfies Bakhvalov's conditions. As a consequence, DiPerna proves the global existence of an entropy solution of the Cauchy problem, provided that the total variation of the initial data is less than $C(p) \tilde{\zeta}$, where $C(p)$ is a constant depending only on the nonlinear function $p(v)$, and $\tilde{\zeta}=\lim _{x \rightarrow \infty}\left\{w\left(U_{0}(x)\right)-z\left(U_{0}(x)\right)\right\}$. In particular, for $p(v)=v^{-\gamma}, 1 \leq \gamma<+\infty$, which clearly satisfies (1.14), one has $C(p) \geq C_{0}$, for some fixed constant $C_{0}>0$, and $\tilde{\zeta}=\frac{1}{\gamma-1} \lim _{x \rightarrow+\infty} v_{0}^{(1-\gamma)}(x)$. In other words, the restriction on the magnitude of the total variation of the initial data is $O\left((\gamma-1)^{-1}\right)$. This shows that the closer $\gamma$ is to 1 the larger the total variation of the initial data can be. For these systems of isentropic gas dynamics, a similar result was also obtained by Nishida and Smoller [16] through different means. Concerning the systems (1.2) satisfying the conditions (1.14), using our periodic formulation based on DiPerna's analysis, we obtain the following result. Set $\zeta(U)=w(U)-z(U)$, and $\bar{\zeta}=\zeta(\bar{U})$. 
Theorem 1.2. Consider problems (1.2)-(1.10) with $p(v)$ satisfying (1.14). Let $\bar{U}$ be as above. There exists and a constant $C(p)$, depending only on $p$, such that if

$$
T V\left(U_{0} \mid[0, \ell)\right) \leq C(p) \bar{\zeta},
$$

then there exists a global periodic entropy solution of (1.2)-(1.10), which belongs to $L^{\infty} \cap B V_{\text {loc }}\left(\mathbb{R} \times \mathbb{R}_{+}\right)$. The solution $U(x, t)$ also satisfies $w(U(x, t)) \leq w_{0}$, $z(U(x, t)) \geq z_{0}$. Furthermore, it has the decay property (1.12).

We also consider the class identified by DiPerna [6], with similar properties to those studied in [1]. We will not give a description of DiPerna's class but instead we will consider the main representative of this class:

$$
\left\{\begin{array}{l}
\rho_{t}+(\rho u)_{x}=0, \\
u_{t}+\left(\frac{1}{2} u^{2}+\frac{\kappa^{2}}{\gamma-1} \rho^{\gamma-1}\right)_{x}=0, \quad \kappa>0,1<\gamma<3,
\end{array}\right.
$$

which is motivated by the isentropic gas dynamics equations for a polytropic gas. Let $U=(\rho, u), F(U)=\frac{1}{2} u^{2}+\frac{\kappa^{2}}{\gamma-1} \rho^{\gamma-1}, \rho>0$, and

$$
z(U)=u-\frac{\kappa}{\beta} \rho^{\beta}, \quad w(U)=u+\frac{\kappa}{\beta} \rho^{\beta}, \quad \beta=\frac{1}{2}(\gamma-1) .
$$

Denote $v=\rho^{-1}$ and consider the initial conditions (1.5)-(1.8) and

$$
z_{0}=\sup _{x \in \mathbb{R}} z\left(U_{0}(x)\right)<w_{0}=\inf _{x \in \mathbb{R}} w\left(U_{0}(x)\right) .
$$

In this paper we also prove the following result concerning the system (1.16).

Theorem 1.3. There exists a global entropy solution of (1.16), (1.5)-(1.8), (1.18), which belongs to $L^{\infty} \cap B V_{\text {loc }}\left(\mathbb{R} \times \mathbb{R}_{+}\right)$and is periodic with period $\ell$. The solution $U(x, t)$ also satisfies $z(U(x, t)) \leq z_{0}, w(U(x, t)) \geq w_{0}$. Moreover, it accomplishes the decay property (1.12).

In Theorems 1.1 1.3 the global boundedness of the periodic solutions is obtained by means of a periodic version of Nishida's modification of the Glimm scheme. The decay property (1.12) is then a consequence of a combination of a theorem in 2 and a compactness theorem in 8 .

In connection with the above theorems, we recall that a theorem in 10] establishes the existence and decay of periodic solutions for a class of strictly hyperbolic genuinely nonlinear $2 \times 2$ systems, including (1.2), with $p^{\prime}(v)<0, p^{\prime \prime}(v)>0$, for $v>0$. The solutions in [10] are obtained by the Glimm method for initial data in $L^{\infty}$ but with very small oscillation. A careful analysis of the asymptotics and the mechanism generating the asymptotic patterns for solutions having the decay property proved in [10] is provided in [4]. Although the classes of systems we consider are slightly less general and the initial data are in $B V_{l o c}$, in many cases our results allow initial data with large oscillation. Finally, we would like to remark that a result analogous to Theorems 1.1 and 1.3 is obtained similarly for the relativistic Euler equations studied by Smoller and Temple in [20], based on a periodic version of the analysis made therein.

The remainder of this paper is organized as follows. In section 2 we address the existence of uniformly bounded periodic solutions for systems (1.2) in Bakhvalov's class, proving the first part of Theorem [1.1] In section 3 we recall the result of DiPerna allowing the inclusion of the systems (1.2), with $p$ satisfying (1.14), in Bakhvalov's class, at least in a neighborhood of a constant state, and we study the 
existence of uniformly bounded periodic solutions for such systems, thus proving the first part of Theorem 1.2. In section 4 we study the existence of uniformly bounded periodic solutions for the systems (1.16), proving the first part of Theorem 1.3. In section 5 we then show how the decay property (1.12) can be achieved in all cases above.

\section{Systems in the Class of Bakhvalov}

We recall that a weak solution of (1.1), 1.5) is a locally integrable function defined over $\mathbb{R} \times[0, \infty)$ satisfying

$$
\iint_{\mathbb{R} \times[0, \infty)} U \phi_{t}+F(U) \phi_{x} d x d t+\int_{\mathbb{R}} U_{0}(x) \phi(x, 0) d x=0,
$$

for any $\phi \in C_{0}^{1}\left(\mathbb{R}^{2}\right)$. Assume that (1.1) is endowed with a strictly convex entropy $\eta(U)$ with entropy flux $q(U)$, that is, $\eta, q$ satisfy

$$
\nabla \eta(U) \nabla F(U)=\nabla q(U) .
$$

We then say that $U(x, t)$ is an entropy solution to (1.1), (1.5) if it is a weak solution of this problem and satisfies

$$
\iint_{\mathbb{R} \times[0, \infty)} \eta(U) \phi_{t}+q(U) \phi_{x} d x d t+\int_{\mathbb{R}} \eta\left(U_{0}(x)\right) \phi(x, 0) d x \geq 0,
$$

for any $\phi \in C_{0}^{1}\left(\mathbb{R}^{2}\right)$, with $\phi \geq 0$.

System (1.1) is strictly hyperbolic if $F^{\prime}(U)$ has real and distinct eigenvalues $\lambda_{1}(U)<\lambda_{2}(U)$. It is genuinely nonlinear if the right eigenvectors of $F^{\prime}(U), r_{1}$ and $r_{2}$, corresponding to $\lambda_{1}$ and $\lambda_{2}$, respectively, satisfy $r_{i} \cdot \nabla \lambda_{i} \neq 0$, for $i=1,2$. A pair of functions $z(U), w(U)$ is a pair of Riemann invariants for (1.1) if $\nabla z \cdot r_{2}=$ $\nabla w \cdot r_{1}=0$ everywhere.

Given a point $U_{0}$, the rarefaction curves by $U_{0}$ are constituted by the points $U$ which can be connected to $U_{0}$ on the right by a rarefaction wave, that is, a solution of (1.1), with initial data

$$
U_{0}(x)= \begin{cases}U_{0}, & x<0 \\ U, & x>0,\end{cases}
$$

continuous for $t>0$ and depending only on $x / t$. For $2 \times 2$ systems there are 2 such curves defined by

$$
\begin{aligned}
& \frac{d U}{d \xi}=\tilde{r}_{i}(U), \quad \xi \geq \lambda_{i}\left(U_{0}\right), \quad i=1,2, \\
& U\left(\lambda_{i}\left(U_{0}\right)\right)=U_{0}
\end{aligned}
$$

where $\tilde{r}_{i}$ are the right eigenvectors normalized by $\nabla \lambda_{i} \cdot \tilde{r}_{i}=1$. In particular, the Riemann invariant $z$ is constant along the rarefaction curves of the second characteristic family while $w$ is constant along those of the first characteristic family.

We also remember that the shock curves by $U_{0}$ consist of the points $U$ satisfying the Rankine-Hugoniot relations

$$
s\left(U-U_{0}\right)=F(U)-F\left(U_{0}\right),
$$


where $s$ is the shock speed. They give rise to solutions of the problem (1.1), (2.4) defined by

$$
U(x, t)= \begin{cases}U_{0}, & x<s t, \\ U, & x>s t .\end{cases}
$$

A shock connecting $U_{r}$ on the right of $U_{l}$ is of the first kind if

$$
\lambda_{1}\left(U_{r}\right)<s<\lambda_{1}\left(U_{l}\right), \quad s<\lambda_{2}\left(U_{r}\right),
$$

and it is of the second kind if

$$
\lambda_{2}\left(U_{r}\right)<s<\lambda_{2}\left(U_{l}\right), \quad s>\lambda_{1}\left(U_{l}\right) .
$$

In this section we consider the strictly hyperbolic genuinely nonlinear system (1.2) for which $U=(v, u)$ and $F(U)=\left(f_{1}(U), f_{2}(U)\right) \equiv(-u, p(v)), \lambda_{1}=-\sqrt{-p^{\prime}(v)}$, $\lambda_{2}=\sqrt{-p^{\prime}(v)}, r_{1}=\left(1, \sqrt{-p^{\prime}(v)}\right)^{\top}, r_{2}=\left(-1, \sqrt{-p^{\prime}(v)}\right)^{\top}$, and $z, w$ given in (1.4) form a pair of Riemann invariants. System (1.2) is endowed with the following strictly convex entropy and associated entropy flux:

$$
\eta(U)=\frac{u^{2}}{2}-\int^{v} p(v) d v, \quad q(U)=u p(v) .
$$

Let $\mathcal{R}$ be the region of the $v, u$-plane given by

$$
\mathcal{R}=\left\{U \in \mathbb{R}^{2}: z(U) \geq z_{0}, w(U) \leq w_{0}\right\},
$$

with $z_{0}$ and $w_{0}$ given in (1.10). Given a point $U_{0} \in \mathcal{R}$, there are curves $R_{i}\left(\alpha ; U_{0}\right)$, $i=1,2$, with $R_{i}\left(0 ; U_{0}\right)=U_{0}$, such that, for $\alpha \geq 0, R_{i}\left(\alpha ; U_{0}\right)$ can be connected to $U_{0}$ on the right by an $i$-rarefaction wave, and for $\alpha<0, R_{i}\left(\alpha ; U_{0}\right)$ can be connected to $U_{0}$ on the right by an $i$-shock wave. We also define the curves $L_{i}\left(\alpha ; U_{0}\right), i=1,2$, satisfying $L_{i}\left(0 ; U_{0}\right)=U_{0}$, such that, for $\alpha \leq 0, L_{i}\left(\alpha ; U_{0}\right)$ can be connected to $U_{0}$ on the left by an $i$-rarefaction wave, and for $\alpha>0, L_{i}\left(\alpha ; U_{0}\right)$ can be connected to $U_{0}$ on the left by an $i$-shock wave. Given points $U_{1}, U_{2} \in \mathcal{R}$, the Riemann problem with left state $U_{1}$ and right state $U_{2}$, that is, the initial value problem for (1.2), with initial data

$$
U_{0}(x)= \begin{cases}U_{1}, & x<0 \\ U_{2}, & x>0\end{cases}
$$

is solved by finding the point $\iota\left(U_{1}, U_{2}\right)=R_{1}\left(\alpha_{1} ; U_{1}\right)=L_{2}\left(\alpha_{2} ; U_{2}\right)$, the intersection of $R_{1}\left(\alpha ; U_{1}\right)$ with $L_{2}\left(\alpha ; U_{2}\right)$. By the properties of the system (1.2), such a point $\iota\left(U_{1}, U_{2}\right)$ always exists, is unique, and belongs to $R$ (see 15, 1]). Then the solution is obtained by connecting $\iota\left(U_{1}, U_{2}\right)=R_{1}\left(U_{1} ; \alpha_{1}\right)$ to $U_{1}$ on the right by a 1 -shock (if $\alpha_{1}<0$ ) or a 1 -rarefaction wave (if $\alpha_{1} \geq 0$ ) and then connecting $U_{2}$ to $\iota\left(U_{1}, U_{2}\right)$ by a 2 -shock (if $\alpha_{2}>0$ ) or a 2-rarefaction (if $\alpha_{2} \leq 0$ ). The properties of the system (1.2), described in 15, 1, also ensure that the solution of the Riemann problem (1.2) and (2.7) takes its values in the region $R$.

The construction of approximate solutions of (1.1), (1.5) proposed by Glimm is well known by now (see, e.g., 9 , 19, 11, 5]). Let $R>0, \bar{U}$ be given by (1.11), and set

$$
\mathcal{V}=\{U \in \mathcal{R}:|U-\bar{U}|<R\} .
$$

Assume the initial data $U_{0}(x)$ satisfies (1.6)-(1.8) and

$$
\operatorname{TV}\left(U_{0} \mid[0, \ell)\right)<\nu(R),
$$


where TV $\left(U_{0} \mid[0, \ell)\right)$ denotes the total variation of $U_{0}$ over one period and $\nu(R)<$ $R$ is to be suitably chosen below. We assume $\nu(R)$ small enough such that, in particular, for any two points $U_{1}, U_{2} \in B(\bar{U} ; \nu(R))$, the open ball centered in $\bar{U}$ with radius $\nu(R)$, satisfying $\left|U_{1}-U_{2}\right|<\nu(R)$, the solution of the Riemann problem (1.1), (2.7) takes its values in $\mathcal{V}$.

Given mesh widths $l=\Delta x, h=\Delta t$, satisfying the Courant-Friedrichs-Lewy condition

$$
A h \leq l, \quad A=\sup \{|\nabla F(U)|: U \in \mathcal{V}\},
$$

we start by taking a piecewise constant approximation of the initial data, say, by setting

$$
U^{h}(x, 0)=\frac{1}{2 l} \int_{m l}^{(m+2) l} U_{0}(x) d x, \quad \text { for } m l<x<(m+2) l, \text { and } m \in \mathbb{Z} \text { is even. }
$$

We define the approximate solution in the strip $\mathbb{R} \times[0, h)$ by solving Riemann problems for each of the discontinuities at the points $(m l, 0), m$ even; condition (2.10) guarantees that these Riemann solutions will not interact in this strip. To define the solution in the next strip $\mathbb{R} \times[h, 2 h)$ we first choose a number $\theta_{1} \in(-1,1)$ and set

$$
U^{h}(x, h)=U^{h}\left(\left(m+1+\theta_{1}\right) l, h-0\right), \quad \text { if } m l<x<(m+2) l, \text { and } m \text { is odd. }
$$

Then we proceed as in the first step solving Riemann problems. The process then continues in the same way for the successive strips $\mathbb{R} \times[k h,(k+1) h), k=2,3, \ldots$, making use of a sampling sequence $\theta_{k} \in(-1,1)$ to define $U^{h}$ as a piecewise constant function at $t=k h$, and then solving Riemann problems. The method can be carried out as long as $U^{h}$ assumes its values in $\mathcal{V}$.

Actually, for systems in Nishida-Bakhvalov's class, the eigenvalues satisfy the a priori bound

$$
\sup \left\{\left|\lambda_{i}(U)\right|: U \in \mathcal{R}\right\} \leq \Lambda, \quad i=1,2,
$$

for some constant $\Lambda>0$. So, the verification of condition (2.10), and hence the possibility of carring out indefinitely the construction of the approximate solutions, would not require the uniform boundedness of the approximate solution, which in general does not hold. Nevertheless, our main concern here is to prove the uniform boundedness of the approximate solutions in the case of periodic initial data, so we will not take advantage of (2.11). As we will see in the next section, for the isentropic gas dynamics systems with $\gamma>1$, the fact that we can make sure that the approximate solutions remain taking their values in a prescribed neighboorhood is a crucial point. On the other hand, this procedure will also be necessary even for the construction of the approximate solutions in the case of systems in DiPerna's class, which will be considered in section 4 for which there is no analogue of (2.11).

From the construction described above we immediately have.

Lemma 2.1. The approximate solution $U^{h}(\cdot, t)$ is periodic with period $\ell$ for any $t>0$ in its interval of existence.

For $U_{1}, U_{2} \in \mathcal{R}$ we define

$$
d\left(U_{1}, U_{2}\right)=\left(z\left(U_{1}\right)-z\left(\iota\left(U_{1}, U_{2}\right)\right)\right)_{+}+\left(w\left(\iota\left(U_{1}, U_{2}\right)\right)-w\left(U_{2}\right)\right)_{+}
$$

with $z, w$ given in (1.4) and $\iota\left(U_{1}, U_{2}\right)$ being, as above, the medium state in the solution of the Riemann problem (1.2), (2.7). As usual, for any real number $y$, we 
denote $(y)_{+}=\max \{y, 0\}$. The special feature of Nishida-Bakhvalov's system (1.2) is summarized in the following lemma whose proof is given in detail in [1].

Lemma 2.2 (Nishida [15], Bakhvalov [1]). Let $U_{1}, U_{2}, U_{3} \in \mathcal{R}$. Then

$$
d\left(U_{1}, U_{3}\right) \leq d\left(U_{1}, U_{2}\right)+d\left(U_{2}, U_{3}\right) .
$$

Moreover, if $U_{2}$ is a value assumed by the solution of the Riemann problem with left state $U_{1}$ and right state $U_{3}$, then equality holds in (2.13).

For a function $U=(v, u) \in B V_{l o c}\left(\mathbb{R}, \mathbb{R}^{2}\right)$, we define

$$
\mu([a, b])=\sup \sum_{k=0}^{N-1} d\left(U\left(x_{k}\right), U\left(x_{k+1}\right)\right), \quad i=1,2,
$$

where the supremum is taken over all partitions $a=x_{0}<x_{1}<\cdots<x_{N}=b$ of the interval $[a, b]$. Lemma $[2.2$ implies that $\mu$ extends to a Radon measure over $\mathbb{R}$. Let $U^{h}=\left(v^{h}, u^{h}\right)$ be an approximate solution constructed by Glimm's method. The periodic version of the Glimm functional, as adapted by Nishida [15], is defined by

$$
F_{p e r}\left(U^{h}(t)\right)=\mu^{h, t}([a, a+\ell)),
$$

where $\mu^{h, t}$ is the measure obtained from $U^{h}(\cdot, t)$ by (2.14) and $a \in \mathbb{R}$ is arbitrary.

A trivial but crucial property of $F_{p e r}$ is stated in the following lemma.

Lemma 2.3. $F_{\text {per }}$ does not depend on a, i.e., $\mu^{h, t}([a, a+\ell))=\mu^{h, t}([0, \ell))$, for any $a \in \mathbb{R}$.

Proof. Immediate from the definition of $F_{\text {per }}$ and Lemma 2.1 .

We take $l=\ell /(2 N)$, for some positive integer $N$ and hold fixed $h / l$ satisfying (2.10). To prove Theorem [1.1 we need the following decisive proposition.

Proposition 2.1. $F_{\text {per }}\left(U^{h}(t)\right)$ is nonincreasing in $t$.

Proof. Since $F_{\text {per }}\left(U^{h}(t)\right)$ is constant for $t \in(k h,(k+1) h), k \in \mathbb{N}$, because of the periodicity (Lemma [2.1) and independence with respect to a (Lemma 2.3), the proof of the proposition reduces to showing that

$$
F_{\text {per }}\left(U^{h}(k h+0)\right) \leq F_{\text {per }}\left(U^{h}(k h-0)\right) .
$$

Let $m_{0} \leq m \leq m_{0}+2 N$, with $m, m_{0} \in\left\{m^{\prime} \in \mathbb{Z} \mid m^{\prime}+k\right.$ is even $\}$, and set $a=\left(m_{0}+\theta_{k}+1\right) l, a_{1}=\left(m+\theta_{k}+1\right) l, a_{2}=\left(m+\theta_{k}+3\right) l$, and then $a+\ell=$ $\left(m_{0}+\theta_{k}+2 N+1\right) l$. Denote $U(x)=U^{h}(x, k h-0)$ and let $\widetilde{U}(x)$ be equal to $U(x)$ if $x \notin\left[a_{1}, a_{2}\right)$ and equal to $U^{h}(x, k h+0)$ if $x \in\left[a_{1}, a_{2}\right)$. A trivial induction reduces the proof of 2.15 to proving that

$$
F_{\text {per }}(\widetilde{U}) \leq F_{\text {per }}(U) .
$$

But Lemma 2.2 implies directly the above inequality. Indeed, if $U_{1}=U^{h}\left(a_{1}, k h-0\right)$, $U_{2}=U^{h}((m+2) l, k h-0)$ and $U_{3}=U^{h}\left(a_{2}, k h-0\right)$, we have

$$
\mu^{h, k h-0}\left(\left[a_{1}, a_{2}\right)\right)=d\left(U_{1}, U_{2}\right)+d\left(U_{2}, U_{3}\right) \geq d\left(U_{1}, U_{3}\right)=\mu^{h, k h+0}\left(\left[a_{1}, a_{2}\right)\right) .
$$

We then arrive at the conclusion that $F_{p e r}\left(U^{h}(t)\right)$ is decreasing in time as desired.

As a direct consequence of Proposition 2.1 we have the following result.

Proposition 2.2. $T V\left(U^{h}(t) \mid[0, \ell)\right)$ is uniformly bounded for $t>0$. 
Proof. The properties of system (1.2) described in 1 imply that the measure $\mu$ is concentrated at the shocks. This is due to the fact that the Riemann invariants $z, w$ decrease across shocks of both characteristic families and increase across rarefaction waves of the corresponding family, being constant across rarefaction waves of the complementary family. It is also a consequence of the properties of system (1.2) described in [1] the fact that across shocks of the first family one has $|[w]| \leq|[z]|$ and for shocks of the second family one has $|[z]| \leq|[w]|$, where as usual the square brackets denote the jump of the enclosed function across the discontinuity. Taking the latter into account and the periodicity we have

$$
\begin{aligned}
\operatorname{TV}\left(U^{h}(t) \mid[0, \ell)\right) & \leq C_{1}\left(\operatorname{TV}\left\{z\left(U^{h}(t) \mid[0, \ell)\right\}+\operatorname{TV}\left\{w\left(U^{h}(t)\right) \mid[0, \ell)\right\}\right)\right. \\
& \leq 2 C_{1}\left(\mathrm { DV } \left\{z\left(U^{h}(t) \mid[0, \ell)\right\}+\mathrm{DV}\left\{w\left(U^{h}(t) \mid[0, \ell)\right\}\right)\right.\right. \\
& \leq 4 C_{1} F_{p e r}\left(U^{h}(t)\right) \leq 4 C_{1} F_{p e r}\left(U^{h}(0)\right) \leq 4 C_{1} C_{2} \operatorname{TV}\left(U^{h}(0) \mid[0, \ell)\right) \\
& \leq 4 C_{1} C_{2} \operatorname{TV}\left(U_{0} \mid[0, \ell)\right),
\end{aligned}
$$

for certain positive constants $C_{1}, C_{2}$ depending only on $F$ and $R$, where DV denotes the decreasing variation. So, we have the uniform boundedness of the variation over one period.

We then impose the following bound on the total variation over one period of the initial data

$$
\max \left\{4 C_{1} C_{2}, 1\right\} \mathrm{TV}\left(U_{0} \mid[0, \ell)\right) \equiv r_{0}<r<R,
$$

for some $0<r<R$. Our goal now is to prove the following proposition:

Proposition 2.3. There exists a sequence of approximate solutions constructed as above which converge almost everywhere to an entropy solution of (1.2), (1.5), such that, given any $t>0$, the approximate solutions $U^{h}$ are defined up to certain time $\bar{t}>t$, provided $h$ is smaller than certain $h_{0}(\bar{t})>0$, and satisfy

$$
\left\|U^{h}(t)-\bar{U}\right\|_{\infty}<R, \quad 0 \leq t<\bar{t}
$$

In order to prove Proposition 2.3 we need a standard result which is a consequence of the uniform bound of the total variation over one period.

Lemma 2.4. The approximate solutions satisfy

$$
\int_{0}^{\ell}\left|U^{h}\left(x, t_{1}\right)-U^{h}\left(x, t_{2}\right)\right| d x \leq C\left(\left|t_{1}-t_{2}\right|+h\right),
$$

for $t_{1}, t_{2}$ belonging to the interval of existence of $U^{h}$, where $C$ depends only on $C_{1}, C_{2}$ and $T V\left(U_{0} \mid[0, \ell)\right)$.

Proof. Follows as the one for the analogous result in [9] (see also [19, 11, 5]). In this case we should also use the periodicity in $x$ of $U^{h}$.

The following lemma is an important step in the proof of Proposition 2.3 .

Lemma 2.5. Let $0<T<(R-r) \ell / 2 C$, with $r, R$ as in (2.16) and $C$ given in (2.18). Assume that for $h$ sufficiently small a sequence of approximate solutions $U^{h}$ is defined up to certain $\bar{t}>N T$, for some $0 \leq N \in \mathbb{Z}$ satisfying both

$$
\begin{aligned}
& \left\|U^{h}(t)-\bar{U}\right\|_{\infty}<R, \quad 0 \leq t \leq N T, \\
& \left|\frac{1}{\ell} \int_{0}^{\ell} U^{h}(x, N T) d x-\bar{U}\right|<\varepsilon<r-r_{0} .
\end{aligned}
$$


Then, if $h<T$, the approximate solutions may be extended up to a certain time $\overline{\bar{t}}>(N+1) T$ satisfying

$$
\left\|U^{h}(t)-\bar{U}\right\|_{\infty}<R, \quad 0 \leq t \leq(N+1) T .
$$

In particular, from (2.8)-(2.9) it follows that the values of $U^{h}(t)$ lie in $\mathcal{V}$, for $0 \leq$ $t \leq(N+1) T$.

Proof. For $N T<t<\bar{t}$, applying Lemma 2.4 we get

$$
\begin{aligned}
\| U^{h}(t) & -\bar{U}\left\|_{\infty} \leq\right\| U^{h}(t)-\frac{1}{\ell} \int_{0}^{\ell} U^{h}(x, t) d x \|_{\infty} \\
& +\left|\frac{1}{\ell} \int_{0}^{\ell} U^{h}(x, t) d x-\frac{1}{\ell} \int_{0}^{\ell} U^{h}(x, N T) d x\right|+\left|\frac{1}{\ell} \int_{0}^{\ell} U^{h}(x, N T) d x-\bar{U}\right| \\
\leq & \operatorname{TV}\left\{U^{h}(t) \mid[0, \ell)\right\}+\frac{C}{\ell}(t-N T+h)+\varepsilon \\
\leq & r_{0}+\varepsilon+\frac{C}{\ell}(t-N T+h) .
\end{aligned}
$$

So, if $T$ and $h$ satisfy the required inequalities, it is possible to prolong $U^{h}$ to an interval $[0, \overline{\bar{t}})$, with $\overline{\bar{t}}>(N+1) T$ verifying the estimate (2.19).

We are now ready to prove Proposition 2.3.

Proof of the Proposition 2.3. By Lemma 2.5 with $N=0$ all $U^{h}$ are defined in the strip $\mathcal{S}_{T}=\mathbb{R} \times[0, T]$. Applying Glimm's consistence proof, we obtain a set

$$
\Theta_{1} \subset \Theta=\prod_{k=1}^{\infty}(-1,1)
$$

with meas $\left(\Theta_{1}\right)=1$ such that any convergent subsequence of $\left\{U^{h}\right\}$, constructed with a sampling sequence belonging to $\Theta_{1}$, converges to a weak solution of (1.2)(1.5), defined in the strip $\mathcal{S}_{T}$. So, we take $\theta^{(1)}=\left\{\theta_{k}^{(1)}\right\}_{k=1}^{\infty} \in \Theta_{1}$ and a subsequence $\left\{h_{s}\right\}, s \in \mathbb{N}$, corresponding to a convergent subsequence $\left\{U^{h_{s}}\right\}$. Let $U$ be the limit of this subsequence, which is defined in the same strip. Since $U$ is a weak solution of (1.2)-(1.5) in $\mathcal{S}_{T}$, and is clearly periodic with period $\ell$, we must have

$$
\frac{1}{\ell} \int_{0}^{\ell} U(x, T) d x=\bar{U}
$$

which is immediate from (1.2) and the periodicity. Let $\varepsilon>0$ satisfy $\varepsilon<r-r_{0}$, with $r, r_{0}$ given by (2.16). By (2.20), given $\theta^{(1)} \in \Theta_{1}$, there exists an $h_{0}=h_{0}\left(\varepsilon, \theta^{(1)}\right)<T$, such that, for all $h_{s}<h_{0}$

$$
\left|\frac{1}{\ell} \int_{0}^{\ell} U^{h_{s}}(x, T) d x-\bar{U}\right|<\varepsilon .
$$

But then, again by Lemma 2.5. with this $\theta^{(1)} \in \Theta_{1}$ fixed, to be used in the strip $[0, T]$, we may extend the approximate solutions $U^{h_{s}}$, with $h_{s}<h_{0}$, to the strip $\mathcal{S}_{2 T}=\mathbb{R} \times[0,2 T]$, applying Glimm's scheme, using any random sequence in the strip $(T, 2 T]$. Now, using again, Glimm's consistence proof, we obtain a $\Theta_{2}$, with meas $\left(\Theta_{2}\right)=1$, such that any convergent subsequence of $\left\{U^{h_{s}}\right\}$, extended to the strip $(T, 2 T]$ by means of a sampling sequence belonging to $\Theta_{2}$, which we still denote by $\left\{U^{h_{s}}\right\}$, converges to a weak solution of (1.2)-(1.5), defined in the strip $\mathcal{S}_{2 T}$. We 
then fix $\theta^{(2)} \in \Theta_{2}$. Then, there exists an $h_{1}=h_{1}\left(\varepsilon, \theta^{(2)}\right)<h_{0}$, such that, for all $h_{s}<h_{1}$,

$$
\left|\frac{1}{\ell} \int_{0}^{\ell} U^{h_{s}}(x, 2 T) d x-\bar{U}\right|<\varepsilon
$$

Again we can apply Lemma 2.5, and for the fixed $\theta^{(1)} \in \Theta_{1}$, which is to be used in the strip $[0, T]$, and $\theta^{(2)} \in \Theta_{2}$, which is to be used in the strip $(T, 2 T]$, the approximate solutions $U^{h_{s}}$, with $h_{s}<h_{1}$, may be extended using Glimm's scheme to the strip $\mathcal{S}_{3 T}=\mathbb{R} \times[0,3 T]$, using any random sequence in the strip $(2 T, 3 T]$. Apply Glimm's consistence proof to obtain a $\Theta_{3}$, with meas $\left(\Theta_{3}\right)=1$, such that any convergent subsequence of $\left\{U^{h_{s}}\right\}$, extended to the strip $(2 T, 3 T]$ by means of a sampling sequence belonging to $\Theta_{3}$, converges to a weak solution of (1.2)(1.5)), defined in the strip $\mathcal{S}_{3 T}$, etc. It is clear that this process may be continued indefinitely. A subsequence satisfying the statement of Proposition 2.3 is then obtained by a standard diagonal process. The fact that the global weak solution so constructed is an entropy solution follows from a well known theorem of Lax [13].

The results in this section clearly imply the existence and uniform boundedness of a periodic entropy solution of (1.2), (1.5) proving Theorem 1.1, except for the last assertion concerning the decay property (1.12). The latter will follow from the results in section 5 .

\section{Periodic solutions For the systems OF ISENTROPIC GAS DYNAMICS, $\gamma>1$}

In this section we discuss the systems (1.2) with $p(v)$ satisfying $p^{\prime}(v)<0, p^{\prime \prime}(v)>$ 0 , for $v>0$, and (1.14) in connection with the analysis of DiPerna in [7]. We first recall a mapping theorem in [7]. Let

$$
\sigma=w+z, \quad \zeta=w-z,
$$

and, for $a \in \mathbb{R}, k \in(0,1]$, consider the regions

$$
W(a, k)=\{(\sigma, \zeta):|\sigma-a| \leq k \zeta\} .
$$

Assume that in (1.2) $p(v)$ satisfies $p^{\prime}(v)<0, p^{\prime \prime}(v)>0$, for $v>0$ and (1.14).

Theorem 3.1 (DiPerna [7]). There exists a 2-parameter family of transformations $T(a, \theta):(z, w) \rightarrow\left(z^{\prime}, w^{\prime}\right), z^{\prime}=\phi(z ; a, \theta), w^{\prime}=\psi(w ; a, \theta),(a, \theta) \in \mathbb{R} \times \mathbb{R}_{+}$, and constants $k, c_{1}, c_{2}(k)$ which have the following property. For sufficiently small $k$, $T(a, \theta)$ maps the shock curves of (1.2) in

$$
H(a, \theta):=W(a, k) \cap\left\{c_{1} / \theta<\zeta<c_{2}(k) / \theta\right\}
$$

onto shock curves which satisfy Bakhvalov's conditions in the $z^{\prime}, w^{\prime}$ variables. Furthermore, $\lim _{k \rightarrow 0} c_{2}(k)=\infty$.

Based on the above theorem the procedures of section 2 can be adapted with slight modifications in order to prove Theorem 1.2, in the following way. Instead of analyzing the growth of the total variation per period and oscillation in the $u, v$ variables it is perhaps more convenient here to work with the $z^{\prime}, w^{\prime}$ variables given by Theorem [3.1. Using the notation in Theorem [3.1] let $\bar{\Sigma}=(\sigma(\bar{U}), \zeta(\bar{U}))$ and $P=T(a, \theta)(\bar{\Sigma})$, with $a=\sigma(\bar{U}), \theta=\left(c_{1}+c_{2}(k)\right) /(2 \bar{\zeta})$, where $\bar{\zeta}=\zeta(\bar{U})$. The choice of $\theta$ is dictated by the analysis in [7]. We may choose $R$ such that the ball 
$S=B(P ; R)$ in the $z^{\prime}, w^{\prime}$ plane satisfies $T^{-1}(a, \theta)(S) \subset H(a, \theta)$. Then restrict the total variation per period of the initial data so that

$$
\mathrm{TV}_{p e r} z_{0}^{\prime}+\mathrm{TV}_{p e r} w_{0}^{\prime} \leq \frac{R}{4}
$$

where $\mathrm{TV}_{\text {per }}$ denotes the total variation per period. As in [7], this amounts to the bound (1.15) for the total variation per period in the $u, v$ variables. For the approximate solutions we obtain the same results as in section 2. Lemma 2.1 is always true and, as a consequence of Theorem 3.1, Lemma 2.2 holds with $z, w$ replaced by $z^{\prime}, w^{\prime}$ in the definition of $d\left(U_{1}, U_{2}\right)$ in (2.12). Lemma 2.3, Propositions 2.1, 2.2. and Lemma 2.4 follow exactly in the same way. Lemma 2.5 and Proposition 2.3 can be easily adapted replacing $\left\|U^{h}(\cdot, t)-\bar{U}\right\|_{\infty}$ by $\left\|T(a, \theta)\left(\Sigma^{h}(\cdot, t)\right)-P\right\|_{\infty}$, with $\Sigma^{h}(x, t)=(\sigma, \zeta)\left(U^{h}(x, t)\right)$, and concluding the corresponding statements. In this way the first part of Theorem 1.2 is proved. We omit further details. The decay property will follow from the discussion in section 5 .

\section{Systems in DiPerna's Class}

In this section we consider the strictly hyperbolic genuine nonlinear systems in DiPerna's class given in (1.16). For this system we have $U=(\rho, u)^{\top}, F(U)=$ $\left(u \rho, \frac{1}{2} u^{2}+\frac{\kappa}{\gamma-1} \rho^{\gamma-1}\right)^{\top}$, the eigenvalues are $\lambda_{1}=u-\kappa \rho^{\beta}, \lambda_{2}=u+\kappa \rho^{\beta}$, where $\beta=(\gamma-1) / 2$, with corresponding eigenvectors $r_{1}=\left(-\rho, \kappa \rho^{\beta}\right)^{\top}, r_{2}=\left(\rho, \kappa \rho^{\beta}\right)^{\top}$, respectively. The Riemann invariants are given by (1.17). System (1.16) is endowed with the following strictly convex entropy and associated entropy flux

$$
\eta(U)=\frac{\kappa^{2}}{(\gamma-1)(\gamma-2)} \rho^{\gamma-1}+\frac{u^{2}}{2}, \quad q(U)=\frac{\kappa^{2}}{(\gamma-2)} \rho^{\gamma-1} u+\frac{u^{3}}{3} .
$$

The analysis here is quite similar to the one in section 2 for the systems in Bakhvalov's class, so we will just give an outline paying more attention to the points where it differs from the latter.

Let $\overline{\mathcal{R}}$ be the region of the $\rho, u$-plane given by

$$
\overline{\mathcal{R}}=\left\{U \in \mathbb{R}^{2}: z(U) \leq z_{0}, w(U) \geq w_{0}\right\},
$$

with $z_{0}$ and $w_{0}$ given in (1.18). Again, given any two points $U_{1}, U_{2} \in \overline{\mathcal{R}}$, the Riemann problem (1.16), (2.7) has a unique solution whose values remain in $\overline{\mathcal{R}}$. The construction of approximate solutions through the Glimm scheme is described in section 2 and Lemma 2.1 holds in general. As in section 3, let

$$
\sigma=w+z=2 u, \quad \zeta=w-z=\frac{2 \kappa}{\beta} \rho^{\beta} .
$$

The properties described in [6] imply that $\sigma$ decreases from left to right across shocks of both characteristic families and increases across rarefaction waves of both families. For $U_{1}, U_{2} \in \overline{\mathcal{R}}$ we define

$$
\bar{d}\left(U_{1}, U_{2}\right)=\left(\sigma\left(U_{1}\right)-\sigma\left(\iota\left(U_{1}, U_{2}\right)\right)\right)_{+}+\left(\sigma\left(\iota\left(U_{1}, U_{2}\right)\right)-\sigma\left(U_{2}\right)\right)_{+}
$$

with $\iota\left(U_{1}, U_{2}\right)$ as in section 2. For $\bar{d}$ we have the following analogue of Lemma 2.2 whose detailed proof is given in [6]. 
Lemma 4.1 (DiPerna [6]). Let $U_{1}, U_{2}, U_{3} \in \overline{\mathcal{R}}$. Then

$$
\bar{d}\left(U_{1}, U_{3}\right) \leq \bar{d}\left(U_{1}, U_{2}\right)+\bar{d}\left(U_{2}, U_{3}\right) .
$$

Moreover, if $U_{2}$ is a value assumed by the solution of the Riemann problem with left state $U_{1}$ and right state $U_{3}$, then equality holds in (4.2).

Again, for a function $U=(v, u) \in B V_{l o c}\left(\mathbb{R}, \mathbb{R}^{2}\right)$, we define

$$
\mu([a, b])=\sup \sum_{k=0}^{N-1} \bar{d}\left(U\left(x_{k}\right), U\left(x_{k+1}\right)\right), \quad i=1,2,
$$

with supremum taken over all partitions $a=x_{0}<x_{1}<\cdots<x_{N}=b$ of the interval $[a, b]$. Lemma 4.1 implies that $\mu$ extends to a Radon measure over $\mathbb{R}$. Let $U^{h}=\left(\rho^{h}, u^{h}\right)$ be an approximate solution constructed by Glimm's method. Again we define

$$
F_{p e r}\left(U^{h}(t)\right)=\mu^{h, t}([a, a+\ell))
$$

where $\mu^{h, t}$ is obtained from $U^{h}(\cdot, t)$ by (4.3) and $a \in \mathbb{R}$ is arbitrary. Clearly, Lemma 2.3 holds here also. Similarly, Proposition 2.1 remains true with identical proof.

Now, in order to obtain the analogue of Proposition 2.2 we need the following result.

Lemma 4.2. Let $(\sigma, \zeta)$ be on an arbitrary shock curve with initial point $\left(\sigma_{0}, \zeta_{0}\right)$. Then

$$
\left|\zeta-\zeta_{0}\right| \leq \frac{1}{\sqrt{\beta}}\left|\sigma-\sigma_{0}\right|
$$

Proof. Following [6], we notice that the Rankine-Hugoniot relations in $\sigma, \zeta$ variables yield

$$
\begin{aligned}
\left(\frac{\sigma-\sigma_{0}}{\zeta-\zeta_{0}}\right)^{2} & =\beta\left(\frac{\zeta+\zeta_{0}}{\zeta^{1 / \beta}+\zeta_{0}^{1 / \beta}}\right)\left(\frac{\zeta^{1 / \beta}-\zeta_{0}^{1 / \beta}}{\zeta-\zeta_{0}}\right) \\
& =\beta \frac{(\epsilon+1)}{(\epsilon-1)} \frac{\left(\epsilon^{1 / \beta}-1\right)}{\left(\epsilon^{1 / \beta}+1\right)} \equiv W(\epsilon),
\end{aligned}
$$

where $\epsilon=\zeta / \zeta_{0}$. Now, as stated in [6], since $0<\beta<1$, we have $\frac{d W}{d \epsilon}>0$, for $0<\epsilon<1$, and $\frac{d W}{d \epsilon}<0$, for $1<\epsilon<\infty$. Since $W(\epsilon) \rightarrow \beta$ as $\epsilon \rightarrow 0$ and $W(\epsilon) \rightarrow \beta$ when $\epsilon \rightarrow+\infty$, we deduce that $W(\epsilon) \geq \beta$, and hence we get (4.4). 
The proof of Proposition 2.2 in the present context now follows as in section 2] the main estimate becoming

$$
\begin{aligned}
\operatorname{TV}\left(U^{h}(t) \mid[0, \ell)\right) & \leq C_{1}\left(\operatorname{TV}\left\{z\left(U^{h}(t) \mid[0, \ell)\right\}+\operatorname{TV}\left\{w\left(U^{h}(t)\right) \mid[0, \ell)\right\}\right)\right. \\
& =2 C_{1}\left(\operatorname{DV}\left\{z\left(U^{h}(t) \mid[0, \ell)\right\}+\operatorname{DV}\left\{w\left(U^{h}(t)\right) \mid[0, \ell)\right\}\right)\right. \\
& \leq 2 C_{1} \sum_{\text {across shocks }}\{|[\sigma]|+|[\zeta]|\} \\
& \leq 2 C_{1}\left(1+\frac{1}{\sqrt{\beta}}\right)\left(\operatorname{DV}\left\{\sigma\left(U^{h}(t) \mid[0, \ell)\right\}\right)\right. \\
& =2 C_{1}\left(1+\frac{1}{\sqrt{\beta}}\right) F\left(U^{h}(t)\right) \leq 2 C_{1}\left(1+\frac{1}{\sqrt{\beta}}\right) F\left(U^{h}(0)\right) \\
& \leq 2\left(1+\frac{1}{\sqrt{\beta}}\right) C_{1} C_{2} \operatorname{TV}\left(U^{h}(0) \mid[0, \ell)\right) \\
& \leq 2\left(1+\frac{1}{\sqrt{\beta}}\right) C_{1} C_{2} \operatorname{TV}\left(U_{0} \mid[0, \ell)\right),
\end{aligned}
$$

for certain positive constants $C_{1}, C_{2}$ depending only on $F$ and $R$. Here, we have used the fact that the Riemann invariants increase from left to right across rarefaction waves, so that the decreasing variation is concentrated on the shocks. We then impose the following bound on the total variation over one period of the initial data

$$
\max \left\{1,2\left(1+\frac{1}{\sqrt{\beta}}\right) C_{1} C_{2}\right\} \operatorname{TV}\left(U_{0} \mid[0, \ell)\right) \equiv r_{0}<r<R,
$$

for some $0<r<R$.

From Proposition 2.2 we again obtain Lemma 2.4 and then Lemma 2.5 Finally, the analogue of Proposition 2.3 in the present context now follows exactly as in the last section for systems in Nishida-Bakhvalov's class. In this way we have proved the existence and uniform boundedness of a periodic entropy solution of (1.16), (1.2), as asserted in Theorem 1.3 To conclude the proof of Theorem 1.3 it remains to prove the decay property (1.12) which will follow from the results in section 5

\section{Dechy of PERIODiC SOlutions}

In this section we show how the decay property of the periodic solutions obtained in sections 1 and 2, as asserted in Theorems 1.11.3 can be achieved. In total, it will be a consequence of their uniform boundedness and the fact that the systems considered are strictly hyperbolic and genuinely nonlinear by applying the results in [2] and [8]. As we will see these decay results show a remarkable interplay between Glimm's [9] and DiPerna's [8] framework, which meet each other through the link provided by the following theorem of [2].

Theorem 5.1 (Chen-Frid [2]). Assume that (1.1) is endowed with a strictly convex entropy, and let $U \in L^{\infty}\left(\mathbb{R} \times \mathbb{R}_{+}\right)$be a periodic entropy solution of (1.1)-(1.5), with period $\ell$. Denote $U^{T}(x, t)=U(T x, T t)$. If the sequence $U^{T}, T \rightarrow \infty$, is precompact in $L_{\text {loc }}^{1}\left(\mathbb{R} \times \mathbb{R}_{+}\right)$, then one has

$$
\text { ess } \lim _{t \rightarrow \infty} \int_{0}^{\ell}|U(x, t)-\bar{U}| d x=0,
$$

where $\bar{U}=\frac{1}{\ell} \int_{0}^{\ell} U_{0}(x) d x$. 
We also recall here the following theorem of DiPerna 8 ].

Theorem 5.2 (DiPerna [8]). Assume (1.1) is a strictly hyperbolic genuinely nonlinear $2 \times 2$ system. Let $U^{T}, T \in I$, for some index set $I$, be a family of entropy solutions of initial value problems for (1.1), which is uniformly bounded in $L^{\infty}\left(\mathbb{R} \times \mathbb{R}_{+}\right)$. Then $U^{T}$ is precompact in $L_{\text {loc }}^{1}\left(\mathbb{R} \times \mathbb{R}_{+}\right)$.

The proof of (1.12) for the periodic solutions constructed in the sections 2 (4) for systems (1.2) and (1.16), now follows easily.

Proof of (1.12). Let $U(x, t)$ be one such periodic solution. From the results in sections [2 4] it follows that $\|U\|_{L^{\infty}\left(\mathbb{R} \times \mathbb{R}_{+}\right)}=M<\infty$. Since $\left\|U^{T}\right\|_{L^{\infty}\left(\mathbb{R} \times \mathbb{R}_{+}\right)}=M$, the sequence is uniformly bounded in $L^{\infty}\left(\mathbb{R} \times \mathbb{R}_{+}\right)$. Now, since both systems are strictly hyperbolic and genuinely nonlinear, by Theorem 5.2 the sequence $U^{T}$ is precompact in $L_{l o c}^{1}\left(\mathbb{R} \times \mathbb{R}_{+}\right)$. But, these systems are also endowed with strictly convex entropy and so Theorem 5.1 directly implies (1.12), which is what we desired to prove.

Remark 5.1. Since its publication, Theorem 5.2 has hardly found application because the assumption of uniform boundedness of the sequence is, in general, too difficult to verify for genuinely nonlinear systems, except in the very special case where the rarefaction curves are straight lines (e.g., [21, 18, 14, 2]). Actually, as far as the author knows, except for this special case the decay results proved here constitute the first applications of Theorem 5.2 which do not assume a priori the uniform boundedness.

Remark 5.2. It is immediate to verify that the methods used here are also applicable to the easier case of $n \times n$ systems with $n$ independent Riemann invariants and coinciding shock and rarefaction curves. We hope that the ideas in this paper can be useful somehow for the solution of the outstanding problem of the global existence of periodic solutions of the $3 \times 3$ system of Euler equations in nonisentropic compressible gas dynamics (see, e.g., [17], 22], [3]).

\section{ACKNOWLEDGEMENTS}

The author wishes to thank Professors James Glimm, Constantine Dafermos and Barbara Keyfitz for their valuable comments on a previous version of the present manuscript. He also gratefully acknowledges the support of CNPq under the grant $352871 / 96-2$.

\section{REFERENCES}

[1] Bakhvalov, N., The existence in the large of a regular solution of a quasilinear hyperbolic system, USSR Comp. Math. and Math. Phys. 10 (1970), 205-219.

[2] Chen, G.-Q., Frid, H., Decay of entropy solutions of nonlinear conservation laws, Arch. Rational Mech. Anal. 146 (1999), 95-127. MR 2000h:35099

[3] Cheverry, C., "Systèmes de Łois de Conservation et Stabilité $B V$ ", Memoires de la Société Mathématique de France 75, Nouvelle série, 1998. MR 2000b:35159

[4] Dafermos, C.M., Large time behavior of periodic solutions of hyperbolic systems of conservation laws, J. Diff. Eqs. 121 (1995), 183-202. MR 96i:35078

[5] Dafermos, C.M., Hyperbolic Conservation Laws in Continuum Physics, Springer-Verlag, 2000. CMP 2000:14

[6] DiPerna, R.J., Global solutions to a class of nonlinear hyperbolic systems of equations, Comm. Pure Appl. Math. 26 (1973), 1-28. MR 48:9125 
[7] DiPerna, R.J., Existence in the large for quasilinear hyperbolic conservation laws Arch. Rat. Mech. Anal. 52 (1973), 244-257. MR 49:3340

[8] DiPerna, R.J., Convergence of approximate solutions to conservation laws, Arch. Rational Mech. Anal. 82 (1983), 27-70. MR 84k:35091

[9] Glimm, J., Solutions in the large for nonlinear hyperbolic systems of equations, Comm. Pure Appl. Math. 18 (1965), 697-715. MR 33:2976

[10] Glimm, J., Lax, P.D., Decay of solutions of systems of nonlinear hyperbolic conservation laws, Memoirs of the Amer. Math. Soc. 101 (1970). MR 42:676

[11] Hörmander, L., Lectures on Nonlinear Hyperbolic Differential Equations, Springer-Verlag, 1997. MR 98e:35103

[12] Lax, P.D., Hyperbolic systems of conservation laws II, Comm. Pure Appl. Math. 10 (1957), 537-566. MR 20:176

[13] Lax, P.D., Shock waves and entropy, In: Contributions to Functional Analysis, ed. E. A. Zarantonello, Academic Press, New York, 1971, pp. 603-634. MR 52:14677

[14] Leveque, R.J. and Temple, B., Stability of Godunov's method for a class of $2 \times 2$ systems of conservation laws, Trans. Amer. Math. Soc. 288 (1985), 115-123. MR 87c:35105

[15] Nishida, T., Global solution for an initial boundary value problem of a quasilinear hyperbolic system, Proc. Japan Acad. 44 (1968), 642-646. MR 38:4821

[16] Nishida, T., Smoller, J., Solutions in the large for some nonlinear hyperbolic conservation laws, Comm. Pure Appl. Math. 26 (1973), 183-200. MR 48:9126

[17] Schochet, S., Glimm scheme for systems with almost planar interactions, Comm. Partial Diff. Eqs., 16 (1991), 1423-1440. MR 93a:35106

[18] Serre, D., Solutions à variations bornées pour certains systèmes hyperboliques de lois de conservations, J. Diff. Eqs. 68 (1987), 137-168. MR 88k:35127

[19] Smoller, J., Shock Waves and Reaction Diffusion Equations, Springer-Verlag, 1983. MR 84d:35002

[20] Smoller, J., Temple, B., Global solutions of the relativistic Euler equations, Commun. Math. Phys. 156 (1993), 67-99. MR 94h:25216

[21] Temple, B., Systems of conservation laws with invariant submanifolds, Trans. Amer. Math. Soc. 280 (1983), 781-795. MR 84m:35080

[22] Temple, J.B., Young, R., Large time stability of sound waves, Comm. Math. Phys. 179 (1996), 417-466. MR 97f:35132

Instituto de Matemática Pura e Aplicada-IMPA, Estrada Dona Castorina, 110, 22460-320 Rio DE JANeiro RJ, BRAsil

E-mail address: hermano@impa.br 